Article

\title{
Improved SP-MCTS-Based Scheduling for Multi-Constraint Hybrid Flow Shop
}

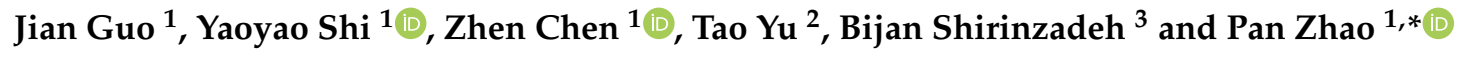 \\ 1 School of Mechanical Engineering, Northwestern Polytechnical University, Xi'an 710072, China; \\ jianguo.NWPU@foxmail.com (J.G.); shiyy@nwpu.edu.cn (Y.S.); changing@mail.nwpu.edu.cn (Z.C.) \\ 2 Xi'an Electronic Engineering Research Institute, Xi'an 710100, China; scor00@163.com \\ 3 Robotics and Mechatronics Research Laboratory, Department of Mechanical and Aerospace Engineering, \\ Monash University, Clayton, VIC 3800, Australia; bijan.shirinzadeh@monash.edu \\ * Correspondence: pan.zhao@nwpu.edu.cn; Tel.: +86-29-8849-2851
}

Received: 1 July 2020; Accepted: 3 August 2020; Published: 8 September 2020

check for updates

\begin{abstract}
As a typical non-deterministic polynomial (NP)-hard combinatorial optimization problem, the hybrid flow shop scheduling problem (HFSSP) is known to be a very common layout in real-life manufacturing scenarios. Even though many metaheuristic approaches have been presented for the HFSSP with makespan criterion, there are limitations of the metaheuristic method in accuracy, efficiency, and adaptability. To address this challenge, an improved SP-MCTS (single-player Monte-Carlo tree search)-based scheduling is proposed for the hybrid flow shop to minimize the makespan considering the multi-constraint. Meanwhile, the Markov decision process (MDP) is applied to transform the HFSSP into the problem of shortest time branch path. The improvement of the algorithm includes the selection policy blending standard deviation, the single-branch expansion strategy and the 4-Rule policy simulation. Based on this improved algorithm, it could accurately locate high-potential branches, economize the resource of the computer and quickly optimize the solution. Then, the parameter combination is introduced to trade off the selection and simulation with the intention of balancing the exploitation and exploration in the search process. Finally, through the analysis of the calculated results, the validity of improved SP-MCTS (ISP-MCTS) for solving the benchmarks is proven, and the ISP-MCTS performs better than the other algorithms in solving large-scale problems.
\end{abstract}

Keywords: hybrid flow shop; scheduling policy; Markov decision process (MDP); single-player Monte-Carlo tree search (SP-MCTS)

\section{Introduction}

The flow shop scheduling problem (FSSP) has been a very active research field, since it was first proposed by Johnson [1]. In the FSSP, a set of $\mathrm{n}$ jobs have to be processed on $\mathrm{m}$ single-machine stages, where each job follows the same route of stages. In production, the FSSP may result in overloading some stages and blocking some jobs [2]. When parallel machines are introduced in some of the stage, the standard flow shop layout becomes a hybrid flow shop (HFS) for these questioners in many real-life manufacturing scenarios. Given its practical interest, the hybrid flow shop scheduling problem (HFSSP) is widely used in today's manufacturing and production systems, such as heavy industry, light industry and other industrial systems. Taking into account the computation complexity, more researchers have conducted in-depth research in this field, since HFSSP has been proved as a non-deterministic polynomial (NP)-hard problem [3]. Simultaneously, production scheduling has been proved to play an important role for improving productivity and responsiveness in the manufacturing system [4]. Therefore, developing more efficient algorithms for this problem is significant. 
Ribas and Ruizad [5] discussed HFSSP in depth, and the methods for solving the HFSSP were divided into the exact algorithm, heuristic method and meta-heuristic algorithm. The exact algorithm used to be the most common solution. In 1970s, the branch and bound algorithm (B\&B) was proposed by Arthanary and Ramaswamy [6] to solve the two-stage HFSSP. Portmann and Vignier [7] combined the $B \& B$ algorithm with the genetic algorithm to solve HFSSP, and the performance of the algorithm was significantly improved. Neron et al. [8] applied energy reasoning and global operations to improve the efficiency of branch definition. Since 1998, many heuristics have been applied to solve HFSSP problems. Gupta and Tunc [9] proposed a heuristic algorithm for solving the minimum completion time of the two-stage HFSSP problem. Kahraman et al. [10] introduced an efficient parallel greedy heuristic algorithm for solving multi-task HFSSP problems. Lin and Liao [11] took a two-step label manufacturing company as an example to minimize the maximum delay of total weight. Heydari and Fakhrzad [12] proposed a heuristic algorithm for minimizing the total time of advance processing and delay processing. In addition, Paternina-Arboleda [13] developed a heuristic method for identifying and continuously solving bottleneck processes. In summary, the heuristic algorithm can quickly construct the scheduling solution, but the quality of the solution is difficult to guarantee.

In recent years, various meta-heuristics have also been developed for solving the HFSSP. Researchers have further studied the application of meta-heuristic algorithms for HFSSP. The taboo algorithm proposed by Nowicki and Smutnick [14] defines the specific neighborhood through critical paths for search optimization. Alaykyran et al. [15] proposed a modified ant colony optimization algorithm to solve HFSSP. Furthermore, Kahraman et al. [16] developed a genetic algorithm which is better than the B\&B method. Liao et al. [17] proposed a particle optimization algorithm, which makes the hybrid discrete particle swarm optimization algorithm and the bottleneck heuristic algorithm to fully explore the bottleneck stage, and combine the simulated annealing algorithm to avoid the local optimal solution. Then, the superiority of the algorithm is illustrated by a comparison of examples. Recently, Marichelvama et al. [18] optimized the HFSSP using the algorithm of Nawaz, Enscore and Ham (NEH) heuristic method, and subsequently applied the CS (cuckoo search) algorithm to quickly search and improve on the basis of the optimized solution. Pan et al. [19] proposed an effective artificial bee colony optimization algorithm, and solved the optimal scheduling solution by hybrid representation method. Li et al. [20] used the variable neighborhood algorithm (HVNS) combined with the chemical reaction optimization algorithm and the distribution estimation algorithm to solve the HFSSP. In the meantime, the algorithm was studied and adjusted to locate the potential region, and search for the optimal solution. Cui and Gu [21] applied the vector notation to model HFSSP, and further proposed an improved discrete artificial bee colony (IDABC) algorithm. Meanwhile, the validity of the IDABC algorithm was verified by using the benchmark problems. Komaki et al. [22] proposed heuristic algorithms and two metaheuristic techniques based on an artificial immune system for a two-stage assembly hybrid flow shop scheduling problem. Zhang and Chen [23] developed a discrete differential evolution (DDE) algorithm with a modified crossover operator to solve larger sized problems. Other algorithms, such as Quantum-inspired immune algorithm (QIA) [24], Artificial immune system (AIS) [25], have also been applied to solve HFSSP. In addition, there are a lot of non-classical methods for solving this kind of large and complex problems. In order to solve the flow shop problem of the minimized makespan, Ramanan et al. [26] proposed a feed forward back propagation neural network to solve the problem. Tkachenko and Izonin $[27,28]$ used neural-like structures based on the geometric transformations model as a universal approximator to implement principles of training and self-training, and thus provided the repeatability of training results and large and small training samples as a satisfactory solution. Gupta et al. [29] investigated the use of these training algorithms as competitive neural network learning tools to minimize makespan in a flow shop. In the studies mentioned above, although the meta-heuristic algorithms were widely used to solve HFSSP, it still has some disadvantages when encountering large problems, such as complex algorithm frameworks, a lack of real-state mapping and incomplete neighborhood structures [30]. 
In order to solve these disadvantages, some scholars adopted machine learning to study HFSSP for achieving an efficient scheduling. Monte-Carlo tree search (MCTS) algorithms heuristically build an asymmetric partial search tree by applying machine learning, and then search through the potential branches to solve the optimal strategy by continuously traversing [31]. Therefore, Chaslot et al. [32] first introduced MCTS for solving production-related problems. A MCTS-based algorithm for the multi-objective flexible job shop scheduling problem was presented by $\mathrm{Wu}$ et al. [33], and it was improved by incorporating the Variable Neighborhood Descent Algorithm and other techniques, like rapid action value, which can estimate the heuristic and transposition table. Chou et al. [34] used the improved MCTS algorithm to solve the multi-objective flexible shop scheduling problem, and search the minimum completion time by the adaptive value game comparison. Furuoka and Matsumoto [35] used an MCTS-based algorithm to find good schedules for a re-entrant scheduling problem. Although good efforts have been made in the above studies, and MCTS is a series of two-person zero-sum game decision-making methods, there are still limitations in the performance and learning efficiency when solving shop floor scheduling problems.

For minimizing the makespan, the HFSSP scheduling approach using improved SP-MCTS (single-player Monte-Carlo tree search) is proposed in this paper. Schadd et al. [36] proposed a new machine learning algorithm, which is named the SP-MCTS algorithm. Schadd et al. [37] applied the algorithm to solve the single-machine puzzle game, and the application algorithm achieved good results in the standardized test. Shimpei et al. [38] verified the effectiveness of the new simulation policy through data experiments, calibrated the relationship between the search method and parameters, and demonstrated the application potential of the SP-MCTS algorithm in actual scheduling.

Through the above review, the improved SP-MCTS (ISP-MCTS) algorithm was successfully applied in the scheduling problems. Therefore, this paper applies ISP-MCTS to solve HFSSP, and the organization of this paper is as follows. In Section 2, the flow shop scheduling problem is introduced, and the mathematical model is established. Then, the improvement scheme of SP-MCTS is introduced in detail in Section 3. In Section 4, the calibration of the algorithm parameters is performed. In Section 5, the experimental results and the comparison are presented. Finally, the conclusions are discussed in Section 6.

\section{Hybrid Flow Shop Scheduling Problem}

The HFSSP is described as follows: a set of $n$ jobs $\mathrm{J}=\{1,2, \ldots, n\}$ must be processed by the $s$-stage, i.e., $\mathrm{O}_{j 1} \rightarrow \mathrm{O}_{j 2}, \ldots, \mathrm{O}_{j s}, \mathrm{~S}=\{1,2, \ldots, s\}$. Each stage $k \in \mathrm{S}$, has $\mathrm{M}_{k} \geq 1$ identical parallel machines, and each job $j$ can be processed in any one of the $\mathrm{M}_{k}$ parallel machines. At the same time, the processing time of the job $j$ in the stage $k\left(\mathrm{O}_{j k}\right)$ with deterministic processing time $\mathrm{P}_{j k}$. Under known the parameters and assumptions, the scheduling policy is solved to minimize the maximum completion time for processing $n$ jobs.

\subsection{Assumption}

The HFSSP problem satisfies the following assumptions:

At the beginning, each job release time is 0 .

Job $j$ can only be processed once in each stage.

Each process of job $j$ can only be processed in one machine, and each machine can only process one job at a time.

There are infinite buffers in the adjacent two stages. Job setup time and the travel time between consecutive stages are included in the processing time $\mathrm{P}_{j s}$ or are ignored.

\subsection{Symbol Definition}

The notation of the HFSSP model is shown as follows:

$\mathrm{P}_{j k}$ is the processing time of job $j$ at stage $k$. 
$\mathrm{D}_{j k}$ is the start processing time of job $j$ at stage $k$.

BM denotes a very large number, the traditional "Big $\mathrm{M}^{\text {". }}$

$\mathrm{x}_{j i k}$ if job $j$ is assigned to machine $i$ at stage $k$, then $\mathrm{x}_{j i k}=1$, otherwise $\mathrm{x}_{j i k}=0$.

$\mathrm{y}_{j j^{\prime} k}$ if job $j$ is processed earlier than $j^{\prime}$ at stage $k$, then $\mathrm{y}_{j j^{\prime} k}=1$, otherwise $\mathrm{y}_{j j^{\prime} k}=0$.

\subsection{Mathematical Model}

Using the above notation, the mathematical relationship of HFSSP is formulated as follows:

$$
\begin{gathered}
\min \left(C_{\max }\right)=\max _{j \in J}\left(D_{j s}+p_{j s}\right) \\
\sum_{i=1}^{m_{k}} x_{j i k}=1, \forall j \in J, k \in S \\
D_{1 j} \geq 0, \forall j \in J \\
D_{k+1, j}-D_{k j} \geq p_{k j}, \forall j \in J, k, k+1 \in S \\
y_{j j^{\prime} k}+y_{j^{\prime} j k} \leq 1, \forall j, j^{\prime} \in J, k \in S \\
D_{k j^{\prime}}-\left(D_{k j}+p_{k j}\right)+B M \cdot\left(3-y_{j j^{\prime} k}-x_{k j i}-x_{k j^{\prime} i}\right) \geq 0, \forall j, j^{\prime} \in J, k \in S, i \in\left\{1,2, \cdots, M_{k}\right\} \\
x_{j i k} \in\{0,1\}, j \in J, k \in S, i \in\left\{1, \cdots, M_{k}\right\} \\
y_{j j^{\prime} k} \in\{0,1\}, j, j^{\prime} \in J, k \in S
\end{gathered}
$$

The objective function (1) is to minimize the maximum completion time. Constraints (2) ensures that each job completes all the processing stages and can only be assigned to one machine in each stage. Equation (3) ensures that the first stage start time of each job is greater than or equal to 0. Equation (4) indicates that when the job is processed in two consecutive stages, the next process can be started after the previous process is completed. Equations (5) and (6) describe the sequential constraints of different jobs in the same machine. When two jobs are processed, the previous job must be finished before the following job can be started. Equations (7) and (8) define the value ranges of decision variables.

\section{ISP-MCTS Algorithm Design}

The MCTS is based on the Monte Carlo simulation to build an asymmetric search tree, and subsequently find the "best" action in the current state [39]. The SP-MCTS algorithm derived from MCTS is proposed to solve HFSSP, and HFSSP is seen as a single-player optimization problem. In the solving process, the processing machines are regarded as starred positions (drop points), and the rules are formulated according to the constraint relationship in the mathematical model. With the goal of maximizing the score, the entire search process includes four phases of selection, expansion, simulation and backpropagation, as shown in Figure 1. 


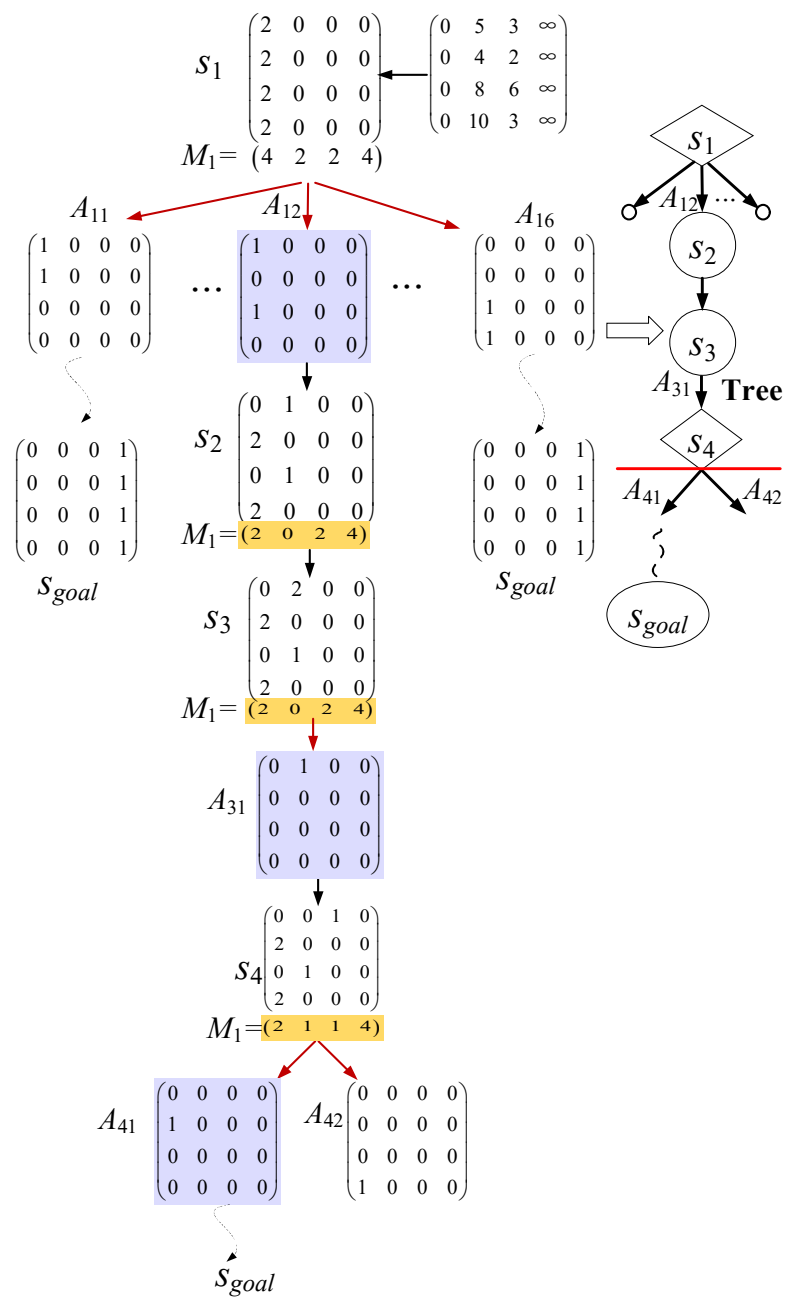

Figure 1. Schematic of the hybrid flow shop scheduling problem (HFSSP) state evolution.

\subsection{HFSSP Model}

According to Markov decision processes (MDP), the model of HFSSP is established [40,41]: (1) $s$ is a series of state matrix of the HFSSP, describing the relationship between the job state and stage. $s_{1}$ and $s_{\text {goal }}$ represent the initial state and completion state in the HFSSP. $s_{1}, s_{2}, \ldots, s_{\text {goal }}$ are used to describe the job production process through various stages. (2) $a$ is the set of scheduling policies in each state $\mathrm{s}$, the scheduling policy sets $a_{1}, a_{2}, \ldots, a_{\mathrm{v}}$ are extracted under each state $\mathrm{s}_{1}, \mathrm{~s}_{2}, \ldots, \mathrm{s}_{\text {goal }}$. A is the current scheduling policy selected from set $a$. Therefore, $\mathrm{A}_{1}, \mathrm{~A}_{2}, \ldots, \mathrm{A}_{\mathrm{v}}$ are the policies corresponding to each state of $s_{1}$ to $s_{\text {goal }}$, selected from the policy sets of $a_{1}, a_{2}, \ldots, a_{\mathrm{v}}$. (3) $\mathrm{T}\left(\mathrm{s}, \mathrm{A}, \mathrm{s}^{\prime}\right)=1$, the scheduling policy $\mathrm{A}$ is selected, and the probability that the state is converted from $\mathrm{s}$ to $\mathrm{s}^{\prime}$ is 1 .

According to the design methodology of the above MDP model, the relationship between the HFSSP field environment and the model state is established. The matrix model $S_{n \times m}$ satisfies the following conditions: (1) $n$ represents the number of jobs, and $m$ represents the number of stages. (2) The first column and the $m$ th column of the matrix are on-line stage and offline stage respectively, the second to the $m-1$ th columns represent the processing stages. (3) The number of jobs being processed must be less than or equal to the number of parallel machines in the stage. (4) The job in the same row is processed according to the sequence of stages, so the job in the next column cannot be started until the job in the current column is completed. (5) For any job, there is $P_{i 1}=0, P_{i m}=\infty$. In Figure $1, S$ is a $4 \times 4$ state matrix, which is the problem of four jobs and four-stage HFSSP. In this matrix, stage 1 and stage 4 are the online stage and offline stage, respectively, and stage 2 and stage 3 have two parallel processing machines. When the state is searched to the $k$ th state matrix, the element 
$\mathrm{s}_{\mathrm{k}(i, j)}=0$ means the job $i$ is not in the machine of the stage $j$, and $\mathrm{s}_{\mathrm{k}(i, j)}=1$ represents that the job $i$ is processing in the stage $j . \mathrm{s}_{\mathrm{k}(i, j)}=2$ represents that the job $i$ has finished processing in the $j$ th stage and waits for scheduling. $a_{k x}$ represents the scheduling policy set $\left(\mathrm{A}_{\mathrm{k} 1}, \mathrm{~A}_{\mathrm{k} 2}, \ldots \mathrm{A}_{\mathrm{ku}}\right)$ in the $\mathrm{s}_{k}$ state. If the element $\mathrm{A}_{k 1(i, j)}=1$, it represents that the job $i$ is dispatched from the machine in the stage $j$ to the processing machine in the stage $j+1$.

As shown in Figure 1, the HFSSP coding model was designed according to the above description. The specific model evolution process is described as follows:

Step Construct the $n \times m$ matrix of the initial state $s_{1}$ and the completion state $s_{\text {goal }}$. In Figure $1, \mathrm{M}$ is 1: the number of parallel machines in each stage, $\mathrm{M}_{1}$ is the number of idle machines in each stage during the process, and the matrix evolves from $\mathrm{s}_{1}$ to $\mathrm{s}_{\text {goal }}$.

Step According to the state of the job and the occupancy of the machine, the available scheduling policy 2: $\quad$ set has $a_{\mathrm{k}}=\left(\mathrm{A}_{\mathrm{k} \gamma}, \mathrm{A}_{\mathrm{k} \beta}, \ldots, \mathrm{A}_{\mathrm{k} \delta}\right)$ during searching for the $k$-floor shop state node $s_{k}$. Meanwhile, the search method is selected on the basis of the traversal times $\mathrm{N}\left(s_{k}\right)$ of the state node $s_{k}$. Details are shown as follows: (1) when $\mathrm{N}\left(\mathrm{s}_{k}\right) \leq \mathrm{P}$ ( $\mathrm{P}$ is the critical value of the simulation times), heuristic rules are applied to select the scheduling policy for searching to the $s_{\text {goal }}$ state; (2) as shown in Figure 1, when $\mathrm{N}(\mathrm{k})>\mathrm{P}$, the selection policy of the SP-MCTS algorithm is used to evaluate the policy set $a_{k}$ and select the scheduling policy $\mathrm{A}_{k}$. After executing strategy $\mathrm{A}_{k}$, it will search from state $s_{k}$ to $s_{k+1}$.

Step If $s_{k+1} \neq s_{\text {goal }}$, step 2 is repeated for state evolution until the $s_{\text {goal }}$.

3:

\subsection{ISP-MCTS Algorithm Optimization Process}

In this paper, the HFSSP scheduling process is constructed as a tree structure. For the SP-MCTS algorithm, there are disadvantages such as poor branch positioning accuracy and slow convergence speed. Therefore, the SP-MCTS algorithm is improved as follows:

\subsubsection{Selection}

The relationship between balanced depth exploration and breadth exploration is constantly explored in the existing state tree nodes, so that each exploration can reach a better solution as far as possible. The UCT (upper confidence bounds applied to trees) algorithm [33] is used to select the scheduling policy, and the improvements are as follows:

$$
\begin{gathered}
Q^{\oplus}(s, a)=Q(s, a)+c \sqrt{2 \ln N(s) / N(s, a)}+\sqrt{\left(\sum q(s, a)^{2}-N(s, a) \times Q(s, a)^{2}+D\right) / N(s, a)} \\
\pi_{U C T}(s)=\underset{a}{\operatorname{argmax} Q^{\oplus}(s, a)}
\end{gathered}
$$

The first two terms of the UCT algorithm in Equation (9) are reserved. N(s) represents the total times of that the state $s$ is accessed, and $\mathrm{N}(s, a)$ represents the times during which the policy $a$ is selected in the state $s . \mathrm{Q}(s, a)$ represents the average score of the policy $a$ that is executed multiple times in state s. Considering a third item here, indicating the deviation obtained after the policy $a$ is executed, where $\sum \mathrm{q}(s, a)^{2}$ represents the sum of the squared results which achieves at the state $s$, constant $D$ is used to ensure that policies with few choices are not underestimated [32]. The purpose of the deviation term without the particularity of the neighborhood is to fully consider the change in range of the score when the policy is selected, and select the policy by using Equation (10) in the end. As shown in Figure 1, taking node $s_{1}$ as an example, when $\mathrm{N}\left(s_{1}\right)>\mathrm{P}$, every policy in the set $\left(\mathrm{A}_{11}, \mathrm{~A}_{12}, \ldots, \mathrm{A}_{16}\right)$ is evaluated according to Equation (9), and the policy is selected on the basis of Equation (10). 


\subsubsection{Expansion}

When $\mathrm{N}(s)>\mathrm{P}$, the branch node is expanded. As shown in Figure 1, if $s_{1}$ node $\mathrm{N}\left(s_{1}\right)>\mathrm{P}$, the scheduling policy $\mathrm{A}_{12}$ is selected, and the node $s_{1}$ is updated to $s_{2}$. If $s_{2}$ is not a node in the tree, $s_{2}$ is added to the tree as a node.

Single-branch node continuous expansion: considering the existence of optional policy uniqueness and state self-updating in HFSSP operation, the single-branch node continuous expansion method is designed. After $s_{2}$ is expanded as shown in Figure 1, the state needs to be self-updated while the job finishes processing, so the state evolves to $s_{3}$. It can be seen that in $s_{3}$, the scheduling policy only has $\mathrm{A}_{31}$. At this time, the node $s_{3}$ is continuously expanded, and then the state evolves to the $s_{4}$, and the number of policies $\left(\mathrm{A}_{41}, \mathrm{~A}_{42}\right)$ is more than 1 . Therefore, node $s_{4}$ is expanded into the tree, and heuristic rules are applied to search the scheduling policy from nodes $s_{4}$ to $s_{\text {goal }}$. As shown in Figure 1, the states $s_{2} \rightarrow s_{4}$ are continuously expanded to avoid multiple meaningless explorations while effectively utilizing computing resources.

\subsubsection{Simulation}

Heuristic rules are used to simulate $s_{\text {goal }}$ from the leaf node $s$. When the tree searches from the $\mathrm{A}_{12}$ branch to the $s_{4}$ leaf node, as shown in Figure 1, $s_{4}$ is used as the simulation initial state. Then, the heuristic rule is applied to search for $s_{\text {goal }}$. Therefore, the root node $s_{1}$ is used as the starting leaf node of the program. Each simulation policy is obtained by different heuristic rule.

According to the literature [19] heuristic rules comparison results, four heuristic rules (4-Rule) are selected as the simulation policy. These four heuristic rules are: (1) $\mathrm{NEH}_{\mathrm{LPT}}(\lambda)$ forward heuristic rule for $\lambda=n \times 50 \%$; (2) $\mathrm{NEH}_{\mathrm{SPTB}}(\lambda)$ forward heuristic rule for $\lambda=n \times 35 \%$; (3) shortest processing time (SPT); and (4) longest processing time (LPT). Before the simulation policy is applied, with probability $\varepsilon$ a random move is played, $\varepsilon=0.005$.

\subsubsection{Backpropagation}

When the simulation is completed, the backpropagation is performed according to the simulation result. Starting from leaf node $s_{4}$, as shown in Figure 1, each child node goes back to its parent node until root node $s_{1}$ is reached. The information is updated as follows:

$$
\begin{gathered}
N(s) \leftarrow N(s)+1 \\
N(s, a) \leftarrow N(s, a)+1 \\
Q(s, a) \leftarrow Q(s, a)+(z-Q(s, a)) / N(s, a) \\
\sum q(s, a)^{2} \leftarrow \sum q(s, a)^{2}+z^{2}
\end{gathered}
$$

Equation (11) represents the total number of times the node $s$ was accessed. Equation (12) records the times that the policy $a$ is executed under the node $s$. Equation (13) updates the average score of the execution policy $a$ at node $s$. Equation (14) is used to calculate the sum of the squared score.

\subsubsection{Evaluation and Policy Set}

The application of ISP-MCT to solve the HFSSP is different from the game-type MCTS problem. In the ISP-MCTS scheduling search, the first iteration time $T_{1}$ is used as the benchmark, and the quality of each subsequent iteration is $\mathrm{T}_{n}-\mathrm{T}_{1}$ as the evaluation score. When $\mathrm{T}_{n}-\mathrm{T}_{1} \leq 0$, the score is $\mathrm{T}_{1}-\mathrm{T}_{n+1}$, and if $\mathrm{T}_{n}-\mathrm{T}_{1}>0$, the score is 0 . Therefore, the optimization purpose is to select the highest scoring policy set. 


\subsection{The Complete Process of ISP-MCTS}

Through the selection policy and the simulation policy, the root node searches from $s_{1}$ to $s_{\text {goal }}$. The algorithm is based on the node average score design selection algorithm and takes the maximum score $\left(\mathrm{T}_{\min }\right)$ for the optimization purpose. Therefore, the pseudo code of the ISP-MCTS for solving HFSSP is described as Algorithm 1.

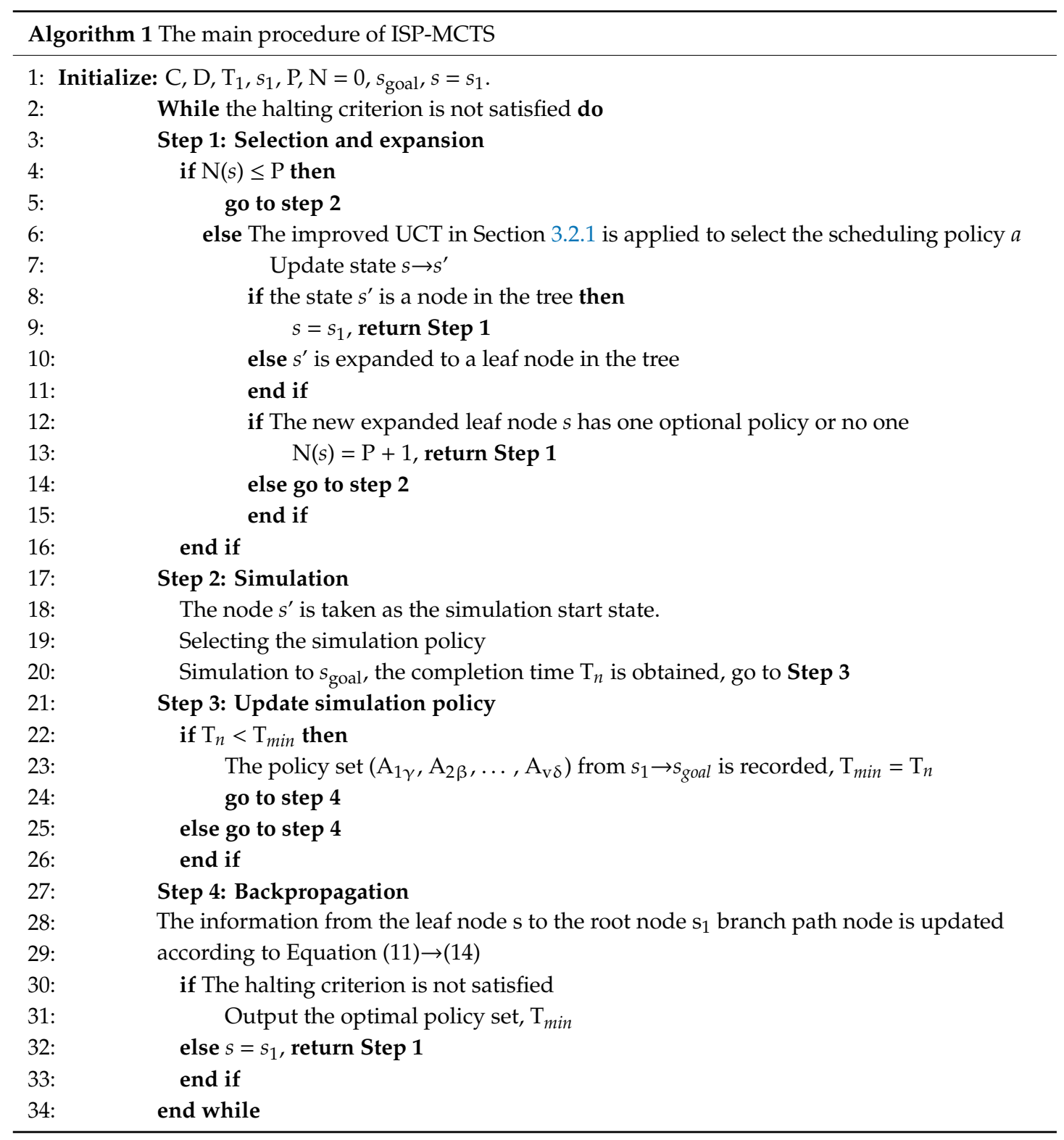

\section{Calibration of the Proposed Algorithms}

\subsection{Simulation Policy Verification $\bar{\alpha}$}

In order to verify the simulation policy mentioned above, a set of instances are generated: $n \in\{20,40,60,80,100\}, s \in\{4,6,8\}$. Ten instances are generated for each combination of $n$ and $s$, and a total of 150 instances are obtained. The processing time is generated from the uniform distribution [1], and the number of parallel machines $\mathrm{M}_{k}$ are generated from the uniform distribution [1,5]. The three simulation policies were constructed in comparison with 4-Rule in Section 3.2.3. The comparison policies are: 4-Rule without continuous expansion (N-Expan), NEH-LPT $(\lambda)$ and random policy (Random). 
Simulation threshold: $\mathrm{P}=4$, taken the combination of expansion and exploration parameters as $\mathrm{C}=0.5$, $\mathrm{D}=10,000$.

These proposed algorithms were coded in MATLAB 2016a, and run on Intel Core i5-3470 3.2 GHz PC with 4 GB memory. The ISP-MCTS limited their run time to $30 n \times s$ ms and ran two fixed replications. The minimum completion time $\mathrm{C}_{\min }$ is the optimal result of all simulation policies under the current instance. The deviation between the final solution of each simulation policy and $C_{\min }$ is as shown in the following Equation (15):

$$
\operatorname{RPI}\left(C_{i}\right)=\left(C_{i}-C_{\min }\right) / C_{\min } \times 100 \%,
$$

In Equation (15), $C_{i}$ is the solution value of the current simulation policy. The average relative percentage increase (ARPI) of the 10 sets of problems under each $n$ and $s$ combination is shown in Table 1. It can be seen that the average deviation of 4-Rule in the four-simulation policy is the smallest, with the average deviation of $0.07 \%$. Meanwhile, the average deviation of the solution of the 4 -Rule simulation policy is better than the simulation policy of the comparison.

Table 1. Comparison results for the RPI values of the four simulation policies.

\begin{tabular}{ccccc}
\hline Problem & 4-Rule & N-Expan & NEH-LPT( $\boldsymbol{\lambda})$ & Random \\
\hline $20 \times 4$ & 0.01 & 0.02 & 4.92 & 12.23 \\
$20 \times 6$ & 0.03 & 0.04 & 4.37 & 13.11 \\
$20 \times 8$ & 0.15 & 0.29 & 5.76 & 14.45 \\
$40 \times 4$ & 0.08 & 0.12 & 4.11 & 10.17 \\
$40 \times 6$ & 0.14 & 0.37 & 4.77 & 13.67 \\
$40 \times 8$ & 0.11 & 0.28 & 5.67 & 15.42 \\
$60 \times 4$ & 0.04 & 0.16 & 4.52 & 12.83 \\
$60 \times 6$ & 0.07 & 0.52 & 4.87 & 14.17 \\
$60 \times 8$ & 0.13 & 0.31 & 5.12 & 17.85 \\
$80 \times 4$ & 0.02 & 0.47 & 2.31 & 11.77 \\
$80 \times 6$ & 0.03 & 0.17 & 7.57 & 13.46 \\
$80 \times 8$ & 0.06 & 0.24 & 6.86 & 15.52 \\
$100 \times 4$ & 0.03 & 0.23 & 3.18 & 12.83 \\
$100 \times 6$ & 0.08 & 0.29 & 3.57 & 17.51 \\
$100 \times 8$ & 0.07 & 0.61 & 6.85 & 21.84 \\
Ave. & 0.07 & 0.275 & 4.963 & 14.455 \\
\hline
\end{tabular}

Notes: RPI: Relative percentage increase. NEH-LPT: the algorithm of Nawaz, Enscore and Ham - longest processing time.

Simulation policies were analyzed using a $40 \times 6$ case instance. Figure 2a shows the relationship between the number of iterations and the node depth. The number of callbacks and average depth of callbacks for each simulation policy are shown in Figure 2b. From Figure 2, NEH-LPT $(\lambda)$ has the greater heuristic depth than the other three simulation policies throughout the iteration. In the iterative process, the number of NEH-LPT $(\lambda)$ callbacks are the least, and the average depth of NEH-LPT $(\lambda)$ callbacks are less than the two algorithms of 4-Rule and N-Expan. Therefore, it can be concluded that the NEH-LPT $(\lambda)$ can easily fall into local minimum. The evaluation positioning is not accurate, and the $\mathrm{N}$-Expan has a high callback frequency in the heuristic process. Therefore, the search depth of the $\mathrm{N}$-Expan is less than 4-Rule. The Random has the least depth of search. From Figure 2b, since 4-Rule has the largest average callback depth, the 4-Rule can be used to accurately locate the branch area within a limited number of callbacks. 


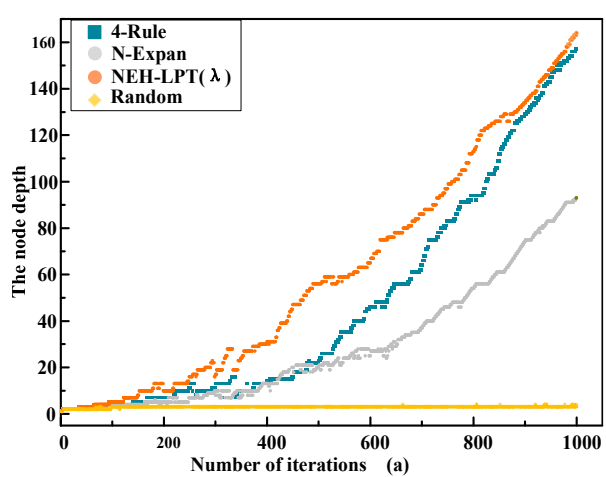

(a)

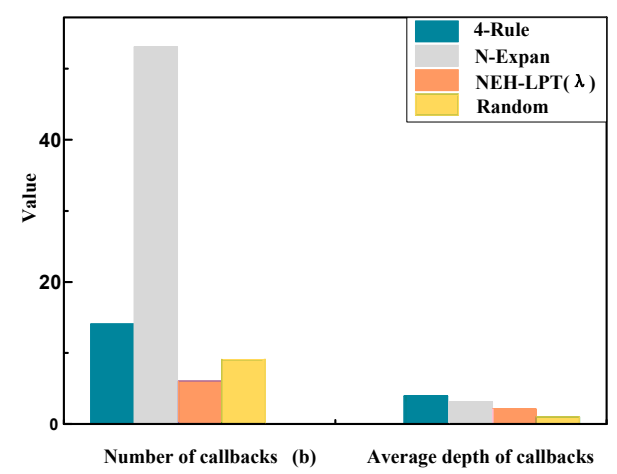

(b)

Figure 2. Schematic of the HFSSP state evolution: (a) the relationship between the number of iterations and the node depth; (b) the number of callbacks and average depth of callbacks for each simulation policy.

A single-factor analysis of variance (ANOVA) was carried out, where the type of simulation policy in Table 2 is considered to be a factor. The 4-Rule and the other three simulation policies are analyzed by multi-comparison method using the least significant difference (LSD) procedure. It indicates that the 4-Rule is significantly better than the other three simulation policies of N-Expan, NEH-LPT $(\lambda)$ and Random. Therefore, the heuristic advantage of the 4-Rule is verified, and the 4-Rule is selected as the simulation policy.

Table 2. The hypothesis tests of 4-Rule with other heuristic schemes.

\begin{tabular}{cccc}
\hline & N-Expan and 4-Rule & NEH-LPT $(\lambda)$ and 4-Rule & Random and 4-Rule \\
\hline$p$-Value & 0.00 & 0.00 & 0.00 \\
Significant? $(p<0.05)$ & Yes & Yes & Yes \\
\hline
\end{tabular}

\subsection{Parameter Setting}

The Benchmark problems [42] and Liao problems [17] were introduced to verify the performance of the ISP-MCT algorithm. The scale of the problems ranged from 10 jobs of 5 stages to 30 jobs of 10 stages. In this section, the parameter combination $(C, D)$ is optimized in three segments according to the search space magnitude. The order of magnitude is $0-10^{5}, 10^{5}-10^{6}$ and $10^{6}-5 \times 10^{6}$. The parameter combination $(0.1,32)$ focuses on the development of potential nodes during the search process. Parameter combination $(1,20,000)$ in the search process considers the exploration ability of unknown regions, and the combination of parameters $(0.5,10,000)$ in the search process takes into account the expansion and exploration capabilities. As shown in Table 3, j15c5d2 is selected for optimization analysis at each stage, and each parameter combination is operated independently for 20 times to obtain an average score. The depth is the distance between the deepest node and the root node, and the average depth is the average of 20 independent running depths. 
Table 3. Results of improved single-player Monte-Carlo tree search (ISP-MCTS) for the different settings.

\begin{tabular}{|c|c|c|c|}
\hline$(\mathrm{C}, \mathrm{D})$ & \multirow{2}{*}{$(0.1,32)$} & \multirow{2}{*}{$(0.5,10,000)$} & \multirow{2}{*}{$(1,20,000)$} \\
\hline $0-10^{5}(\mathrm{j} 15 \mathrm{c} 5 \mathrm{~d} 2)(\sim 10 \mathrm{~s})$ & & & \\
\hline Average completion time & 84.83 & 85.14 & 88.27 \\
\hline Standard deviation & 0.53 & 1.27 & 2.50 \\
\hline Average depth & 57 & 19 & 11 \\
\hline \multicolumn{4}{|l|}{$10^{5}-10^{6}(\mathrm{j} 30 \mathrm{c} 5 \mathrm{e} 4)(\sim 100 \mathrm{~s})$} \\
\hline Average completion time & 570.9 & 567.3 & 581.1 \\
\hline Standard deviation & 3.51 & 2.77 & 4.73 \\
\hline Average depth & 207 & 73 & 14 \\
\hline \multicolumn{4}{|l|}{$10^{6}-5 \times 10^{6}(\mathrm{j} 30 \mathrm{c} 5 \mathrm{e} 1)(\sim 200 \mathrm{~s})$} \\
\hline Average completion time & 482.7 & 465.3 & 480.5 \\
\hline Standard deviation & 3.56 & 2.72 & 3.21 \\
\hline Average depth & 289 & 98 & 27 \\
\hline
\end{tabular}

As shown in Table 3, in the order of magnitude of $10^{5}-10^{6}, 10^{6}-5 \times 10^{6}$ within the specified time limit, the ISP-MCTS obtains the minimum average completion time and standard deviation, when the parameter combination is $(0.1,32)$. When applying the parameter combination $(0.1,32)$, the ISP-MCTS finds the minimum average completion time. The average depth of the search tree at the parameter combination $(0.1,32)$ is 57 , then most of the nodes generated in the scheduling of the 15 jobs of 5 stages have been extended to the tree by the ISP-MCTS. Compared with the other two sets of parameters $(0.5$, $10,000)$ and $(1,20,000)$, the parameter combination $(0.1,32)$ expands the node deeper.

In the order of magnitude $10^{5} \sim 10^{6}, 10^{6} \sim 5 \times 10^{6}$ within the specified time limit, when the parameter combination is $(0.5,10,000)$, the ISP-MCTS finds that the average minimum completion time is optimal, and the algorithm stability is better than other combinations.

With the increasing magnitude and search time, the exploratory parameter combination advantage becomes more and more obvious. Therefore, at the order of magnitude of $10^{6}-5 \times 10^{6}$, the exploration parameter $(1,20,000)$ was significantly better than the parameter combination $(0.1,32)$.

It can be observed from the above that the order of magnitude is $0-10^{5}$ within the specified time limit. When the parameter combination is $(0.1,32)$, the ISP-MCTS algorithm has the highest solution quality and the best stability. Therefore, when the order of magnitude is $10^{5} \sim 10^{6}$ or $10^{6} \sim 5 \times 10^{6}$, the algorithm selects the parameter combination $(0.5,10,000)$.

\section{Calculation Results and Analysis}

According to the machine layout, the Benchmark problems is divided into two groups: (1) 47 a and $\mathrm{b}$ problems; and (2) $30 \mathrm{c}$ and d problems. The ISP-MCTS algorithm was programmed in MATLAB 2016a and run on a central processing unit (CPU)-i5-3470(3.20GHZ) with 4.0 GB Main Memory. Six algorithms HVNS [20], IDABC [21], particle swarm optimization (PSO) [17], AIS [25], genetic algorithm (GA) [16] and $\mathrm{B} \& \mathrm{~B}[8]$ are selected as comparison algorithms. The calculation results of the above comparison algorithm are obtained from the source literature. The HVNS algorithm sets the maximum running time to $100 \mathrm{~s}$ and takes the minimum completion time of 20 independent results under the same conditions. The IDABC algorithm also limits their runtime to $1600 \mathrm{~s}$ and runs 20 times independently. The maximum running time of the algorithms B\&B, AIS, GA and PSO in the literature is limited to $1600 \mathrm{~s}$. The minimum $C_{\max }$ is obtained as the final solution by independently operating 20 times under the same conditions, and thus the CPU time corresponding to $C_{\max }$ is recorded for each problem. The performance of each algorithm is compared by "\%Deviation", and the deviation between the lower bound LB and each algorithm $C_{\max }$ is obtained by using Equation (16), as shown in Tables 4 and 5. The statistical analysis results are shown in Table 6:

$$
\text { \%deviation }=\left(\frac{C_{\max }-L B}{L B}\right) \times 100 \%
$$


Table 4. Comparison results for the type a and type b problems.

\begin{tabular}{|c|c|c|c|c|c|c|c|c|c|c|c|c|c|c|c|}
\hline \multirow{2}{*}{ Problem } & \multirow{2}{*}{ LB } & \multicolumn{2}{|c|}{ ISP-MCTS } & \multicolumn{2}{|c|}{ IDABC } & \multicolumn{2}{|c|}{ HVNS } & \multicolumn{2}{|c|}{ PSO } & \multicolumn{2}{|c|}{ GA } & \multicolumn{2}{|c|}{ AIS } & \multicolumn{2}{|c|}{ B\&B } \\
\hline & & $\mathrm{C}_{\max }$ & CPU & $\mathrm{C}_{\max }$ & CPU & $C_{\max }$ & CPU & $\mathrm{C}_{\max }$ & CPU & $\mathrm{C}_{\max }$ & CPU & $\mathrm{C}_{\max }$ & CPU & $\mathrm{C}_{\max }$ & CPU \\
\hline j10c5a2 & 88 & 88 & 0.003 & 88 & 0.002 & 88 & 0.004 & 88 & 0.002 & 88 & 0.000 & 88 & 1 & 88 & 13 \\
\hline j10c5a3 & 117 & 117 & 0.003 & 117 & 0.003 & 117 & 0.004 & 117 & 0.002 & 117 & 0.000 & 117 & 1 & 117 & 7 \\
\hline j10c5a4 & 121 & 121 & 0.002 & 121 & 0.003 & 121 & 0.002 & 121 & 0.003 & 121 & 0.015 & 121 & 1 & 121 & 6 \\
\hline j10c5a5 & 122 & 122 & 0.004 & 122 & 0.005 & 122 & 0.003 & 122 & 0.013 & 122 & 0.000 & 122 & 1 & 122 & 11 \\
\hline j10c5a6 & 110 & 110 & 0.022 & 110 & 0.009 & 110 & 0.024 & 110 & 0.174 & 110 & 0.015 & 110 & 4 & 110 & 6 \\
\hline j10c5b1 & 130 & 130 & 0.003 & 130 & 0.003 & 130 & 0.003 & 130 & 0.003 & 130 & 0.000 & 130 & 1 & 130 & 13 \\
\hline j10c5b2 & 107 & 107 & 0.003 & 107 & 0.004 & 107 & 0.003 & 107 & 0.003 & 107 & 0.000 & 107 & 1 & 107 & 6 \\
\hline j10c5b3 & 109 & 109 & 0.003 & 109 & 0.003 & 109 & 0.003 & 109 & 0.012 & 109 & 0.000 & 109 & 1 & 109 & 9 \\
\hline j10c5b4 & 122 & 122 & 0.003 & 122 & 0.003 & 122 & 0.004 & 122 & 0.025 & 122 & 0.000 & 122 & 2 & 122 & 6 \\
\hline j10c5b5 & 153 & 153 & 0.003 & 153 & 0.003 & 153 & 0.003 & 153 & 0.001 & 153 & 0.000 & 153 & 1 & 153 & 6 \\
\hline j10c5b6 & 115 & 115 & 0.003 & 115 & 0.004 & 115 & 0.002 & 115 & 0.001 & 115 & 0.000 & 115 & 1 & 115 & 11 \\
\hline j10c10a1 & 139 & 139 & 0.031 & 139 & 0.009 & 139 & 0.227 & 139 & 0.055 & 139 & 0.015 & 139 & 1 & 139 & 41 \\
\hline j10c10a2 & 158 & 158 & 0.337 & 158 & 0.009 & 158 & 0.354 & 158 & 0.87 & 158 & 0.125 & 158 & 18 & 158 & 21 \\
\hline j10c10a3 & 148 & 148 & 0.036 & 148 & 0.008 & 148 & 0.225 & 148 & 0.017 & 148 & 0.047 & 148 & 1 & 148 & 58 \\
\hline j10c10a4 & 149 & 149 & 0.010 & 149 & 0.007 & 149 & 0.007 & 149 & 0.085 & 149 & 0.141 & 149 & 2 & 149 & 21 \\
\hline j10c10a5 & 148 & 148 & 0.006 & 148 & 0.006 & 148 & 0.006 & 148 & 0.102 & 148 & 0.000 & 148 & 1 & 148 & 36 \\
\hline j10c10a6 & 146 & 146 & 0.282 & 146 & 0.008 & 146 & 0.264 & 146 & 0.239 & 146 & 0.156 & 146 & 4 & 146 & 20 \\
\hline j10c10b1 & 163 & 163 & 0.006 & 163 & 0.007 & 163 & 0.006 & 163 & 0.013 & 163 & 0.000 & 163 & 1 & 163 & 36 \\
\hline j10c10b2 & 157 & 157 & 0.114 & 157 & 0.009 & 157 & 0.104 & 157 & 0.221 & 157 & 0.131 & 157 & 1 & 157 & 66 \\
\hline j10c10b3 & 169 & 169 & 0.007 & 169 & 0.007 & 169 & 0.006 & 169 & 0.014 & 169 & 0.000 & 169 & 1 & 169 & 19 \\
\hline j10c10b4 & 159 & 159 & 0.012 & 159 & 0.005 & 159 & 0.068 & 159 & 0.021 & 159 & 0.015 & 159 & 1 & 159 & 20 \\
\hline j10c10b5 & 165 & 165 & 0.007 & 165 & 0.007 & 165 & 0.005 & 165 & 0.037 & 165 & 0.016 & 165 & 1 & 165 & 33 \\
\hline j10c10b6 & 165 & 165 & 0.008 & 165 & 0.006 & 165 & 0.005 & 165 & 0.056 & 165 & 0.016 & 165 & 1 & 165 & 34 \\
\hline j15c5a1 & 178 & 178 & 0.007 & 178 & 0.016 & 178 & 0.006 & 178 & 0.060 & 178 & 0.031 & 178 & 1 & 178 & 18 \\
\hline j15c5a2 & 165 & 165 & 0.005 & 165 & 0.018 & 165 & 0.005 & 165 & 0.005 & 165 & 0.015 & 165 & 1 & 165 & 35 \\
\hline j15c5a3 & 130 & 130 & 0.008 & 130 & 0.017 & 130 & 0.005 & 130 & 0.006 & 130 & 0.015 & 130 & 1 & 130 & 34 \\
\hline j15c5a4 & 156 & 156 & 0.010 & 156 & 0.019 & 156 & 0.005 & 156 & 0.013 & 156 & 0.015 & 156 & 2 & 156 & 21 \\
\hline j15c5a5 & 164 & 164 & 0.005 & 164 & 0.016 & 164 & 0.003 & 164 & 0.004 & 164 & 0.046 & 164 & 1 & 164 & 34 \\
\hline j15c5a6 & 178 & 178 & 0.007 & 178 & 0.016 & 178 & 0.005 & 178 & 0.006 & 178 & 0.032 & 178 & 1 & 178 & 38 \\
\hline j15c5b1 & 170 & 170 & 0.007 & 170 & 0.012 & 170 & 0.005 & 170 & 0.003 & 170 & 0.015 & 170 & 1 & 170 & 16 \\
\hline $\mathrm{j} 15 \mathrm{c} 5 \mathrm{~b} 2$ & 152 & 152 & 0.005 & 152 & 0.015 & 152 & 0.003 & 152 & 0.005 & 152 & 0.015 & 152 & 1 & 152 & 25 \\
\hline j15c5b3 & 157 & 157 & 0.006 & 157 & 0.014 & 157 & 0.006 & 157 & 0.030 & 157 & 0.015 & 157 & 1 & 157 & 15 \\
\hline j15c5b4 & 147 & 147 & 0.010 & 147 & 0.017 & 147 & 0.007 & 147 & 0.000 & 147 & 0.015 & 147 & 1 & 147 & 37 \\
\hline j15c5b5 & 166 & 166 & 0.100 & 166 & 0.020 & 166 & 0.088 & 166 & 0.086 & 166 & 0.016 & 166 & 2 & 166 & 20 \\
\hline j15c5b6 & 175 & 175 & 0.007 & 175 & 0.018 & 175 & 0.004 & 175 & 0.016 & 175 & 0.015 & 175 & 1 & 175 & 23 \\
\hline j15c10a1 & 236 & 236 & 0.011 & 236 & 0.033 & 236 & 0.008 & 236 & 0.018 & 236 & 0.015 & 236 & 1 & 236 & 40 \\
\hline j15c10a2 & 200 & 200 & 0.120 & 200 & 0.048 & 200 & 0.339 & 200 & 0.214 & 200 & 0.015 & 200 & 30 & 200 & 154 \\
\hline j15c10a3 & 198 & 198 & 0.130 & 198 & 0.032 & 198 & 0.313 & 198 & 0.171 & 198 & 0.063 & 198 & 4 & 198 & 45 \\
\hline j15c10a4 & 225 & 225 & 0.070 & 225 & 0.034 & 225 & 0.212 & 225 & 0.072 & 225 & 0.031 & 225 & 12 & 225 & 78 \\
\hline j15c10a5 & 182 & 182 & 0.011 & 182 & 0.036 & 182 & 0.008 & 182 & 0.509 & 182 & 0.016 & 182 & 2 & 183 & c \\
\hline j15c10a6 & 200 & 200 & 0.052 & 200 & 0.033 & 200 & 0.024 & 200 & 0.468 & 200 & 0.031 & 200 & 2 & 200 & 44 \\
\hline j15c10b1 & 222 & 222 & 0.026 & 222 & 0.048 & 222 & 0.009 & 222 & 0.017 & 222 & 0.031 & 222 & 3 & 222 & 70 \\
\hline j15c10b2 & 187 & 187 & 0.009 & 187 & 0.044 & 187 & 0.008 & 187 & 0.012 & 187 & 0.047 & 187 & 1 & 187 & 80 \\
\hline j15c10b3 & 222 & 222 & 0.009 & 222 & 0.039 & 222 & 0.008 & 222 & 0.007 & 222 & 0.015 & 222 & 1 & 222 & 80 \\
\hline j15c10b4 & 221 & 221 & 0.009 & 221 & 0.037 & 221 & 0.008 & 221 & 0.007 & 221 & 0.016 & 221 & 1 & 221 & 84 \\
\hline j15c10b5 & 200 & 200 & 0.109 & 200 & 0.048 & 200 & 0.045 & 200 & 0.135 & 200 & 0.094 & 200 & 1 & 200 & 84 \\
\hline j15c10b6 & 219 & 219 & 0.010 & 219 & 0.037 & 219 & 0.009 & 219 & 0.006 & 219 & 0.031 & 219 & 1 & 219 & 67 \\
\hline AVE & & & 0.035 & & 0.017 & & 0.052 & & 0.082 & & 0.028 & & 2.574 & & 35.674 \\
\hline
\end{tabular}

Notes: CPU: The CPU time of the algorithm in seconds. ISP-MCTS: Improved single-player Monte-Carlo tree search. IDABC: Improved discrete artificial bee colony algorithm. HVNS: Hybrid variable neighborhood search. PSO: Particle swarm optimization. GA: Genetic algorithm. AIS: Artificial immune system. B\&B: Branch-and-bound algorithm. $\mathrm{GA}_{\mathrm{R}}$ : Genetic algorithm (Ruiz, R). ISA: Improved simulated annealing. $A_{B C}$ : Artificial bee colony algorithm with permutation. 
Table 5. Comparison results for type c and d problems.

\begin{tabular}{|c|c|c|c|c|c|c|c|c|c|c|c|c|c|c|c|}
\hline \multirow{2}{*}{ Problem } & \multirow{2}{*}{ LB } & \multicolumn{2}{|c|}{ ISP-MCTS } & \multicolumn{2}{|c|}{ IDABC } & \multicolumn{2}{|c|}{ HVNS } & \multicolumn{2}{|c|}{ PSO } & \multicolumn{2}{|c|}{ GA } & \multicolumn{2}{|c|}{ AIS } & \multicolumn{2}{|c|}{ B\&B } \\
\hline & & $\mathrm{C}_{\max }$ & CPU & $\mathrm{C}_{\max }$ & CPU & $\mathrm{C}_{\max }$ & CPU & $\mathrm{C}_{\max }$ & CPU & $\mathrm{C}_{\max }$ & CPU & $\mathrm{C}_{\max }$ & CPU & $\mathrm{C}_{\max }$ & CPU \\
\hline j10c5c1 & 68 & 68 & 0.275 & 68 & 0.017 & 68 & 0.792 & 68 & 0.332 & 68 & 0.031 & 68 & 32 & 68 & 28 \\
\hline j10c5c2 & 74 & 74 & 0.633 & 74 & 0.055 & 74 & 0.752 & 74 & 0.535 & 74 & 0.016 & 74 & 4 & 74 & 19 \\
\hline j10c5c3 & 71 & 71 & 0.752 & 72 & a & 72 & $0.141(\mathrm{a})$ & 71 & 36.997 & 71 & 0.016 & 72 & a & 71 & 240 \\
\hline j10c5c4 & 66 & 66 & 0.161 & 66 & 0.005 & 66 & 0.631 & 66 & 0.215 & 66 & 0.031 & 66 & 3 & 66 & 1017 \\
\hline j10c5c5 & 78 & 78 & 0.382 & 78 & 0.004 & 78 & 0.129 & 78 & 0.122 & 78 & 0.094 & 78 & 14 & 78 & 42 \\
\hline j10c5c6 & 69 & 69 & 0.527 & 69 & 0.004 & 69 & 0.226 & 69 & 0.405 & 69 & 0.000 & 69 & 12 & 69 & 4865 \\
\hline j10c5d1 & 66 & 66 & 0.268 & 66 & 0.005 & 66 & 0.148 & 66 & 0.185 & 66 & 0.046 & 66 & 5 & 66 & 6490 \\
\hline j10c5d2 & 73 & 73 & 0.271 & 73 & 0.069 & 73 & 0.142 & 73 & 1.158 & 73 & 0.110 & 73 & 31 & 73 & 2617 \\
\hline j10c5d3 & 64 & 64 & 0.277 & 64 & 0.007 & 64 & 0.157 & 64 & 0.098 & 64 & 0.015 & 64 & 15 & 64 & 481 \\
\hline j10c5d4 & 70 & 70 & 0.895 & 70 & 0.003 & 70 & 0.675 & 70 & 0.337 & 70 & 0.000 & 70 & 5 & 70 & 393 \\
\hline j10c5d5 & 66 & 66 & 1.576 & 66 & 0.009 & 66 & 1.382 & 66 & 0.515 & 66 & 0.031 & 66 & 1446 & 66 & 1627 \\
\hline j10c5d6 & 62 & 62 & 0.276 & 62 & 0.009 & 62 & 0.135 & 62 & 0.383 & 62 & 0.062 & 62 & 8 & 62 & 6861 \\
\hline j10c10c1 & 113 & 114 & $2.822(\mathrm{a})$ & 115 & a & 115 & $0.571(\mathrm{a})$ & 115 & a & 115 & a & 115 & a & 127 & $\mathrm{c}$ \\
\hline j10c10c2 & 116 & 117 & $0.414(\mathrm{a})$ & 119 & a & 117 & $0.448(a)$ & 117 & a & 117 & a & 119 & a & 116 & 1100 \\
\hline j10c10c3 & 98 & 108 & $2.706(\mathrm{a})$ & 116 & a & 116 & $0.698(a)$ & 116 & a & 116 & a & 116 & a & 133 & c \\
\hline j10c10c4 & 103 & 112 & $2.315(\mathrm{a})$ & 120 & a & 120 & $0.461(a)$ & 120 & a & 120 & a & 120 & a & 135 & c \\
\hline j10c10c5 & 121 & 125 & 2.831(a) & 125 & a & 125 & $2.125(\mathrm{a})$ & 125 & a & 125 & a & 126 & a & 145 & c \\
\hline j10c10c6 & 97 & 105 & $2.663(\mathrm{a})$ & 106 & a & 106 & $0.343(\mathrm{a})$ & 106 & a & 106 & a & 106 & a & 112 & c \\
\hline j15c5c1 & 85 & 85 & 1.179 & 85 & 0.127 & 85 & 1.128 & 85 & 4.205 & 85 & 0.031 & 85 & 774 & 85 & 2131 \\
\hline $\mathrm{j} 15 \mathrm{c} 5 \mathrm{c} 2$ & 90 & 90 & 0.785 & 90 & 27 & 90 & 0.491 & 90 & 1198 & 91 & $\mathrm{a}$ & 91 & $\mathrm{a}$ & 90 & 184 \\
\hline j15c5c3 & 87 & 87 & 0.511 & 87 & 0.048 & 87 & 0.502 & 87 & 2.398 & 87 & 0.109 & 87 & 16 & 87 & 202 \\
\hline j15c5c4 & 89 & 89 & 0.653 & 89 & 0.038 & 89 & 0.569 & 89 & 2.208 & 89 & 0.000 & 89 & 317 & 90 & c \\
\hline j15c5c5 & 73 & 74 & $2.197(a)$ & 74 & a & 74 & $1.968(\mathrm{a})$ & 74 & a & 75 & a & 74 & a & 84 & c \\
\hline j15c5c6 & 91 & 91 & 0.370 & 91 & 0.027 & 91 & 0.180 & 91 & 0.191 & 91 & 0.047 & 91 & 19 & 91 & 57 \\
\hline j15c5d1 & 167 & 167 & 0.005 & 167 & 0.020 & 167 & 0.004 & 167 & 0 & 167 & 0.015 & 167 & 1 & 167 & 24 \\
\hline $\mathrm{j} 15 \mathrm{c} 5 \mathrm{~d} 2$ & 82 & 84 & $1.133(\mathrm{a})$ & 84 & a & 84 & $0.915(\mathrm{a})$ & 84 & a & 84 & $\mathrm{a}$ & 84 & a & 85 & c \\
\hline $\mathrm{j} 15 \mathrm{c} 5 \mathrm{~d} 3$ & 77 & 82 & $2.171(\mathrm{a})$ & 82 & a & 82 & $1.356(\mathrm{a})$ & 82 & a & 83 & a & 83 & a & 96 & c \\
\hline j15c5d4 & 61 & 84 & $0.672(a)$ & 84 & a & 84 & $1.325(\mathrm{a})$ & 84 & a & 84 & a & 84 & a & 101 & c \\
\hline j15c5d5 & 67 & 79 & 1.559 (a) & 79 & a & 79 & $0.585(\mathrm{a})$ & 79 & a & 80 & a & 80 & a & 97 & c \\
\hline j15c5d6 & 79 & 81 & 1.683(a) & 81 & a & 81 & $1.123(\mathrm{a})$ & 81 & a & 82 & a & 82 & $\mathrm{a}$ & 87 & c \\
\hline AVE & & & 0.544 & & 1.615 & & 0.473 & & 73.43 & & 0.038 & & 168.88 & & 455.23 \\
\hline
\end{tabular}

Table 6. Performance summary of the different algorithms.

\begin{tabular}{ccccc}
\hline \multirow{2}{*}{ Algorithm } & \multicolumn{2}{c}{ a and b Problems } & \multicolumn{2}{c}{$\mathbf{c}$ and d Problems } \\
\cline { 2 - 5 } & Solved & Deviation & Solved & Deviation \\
\hline ISP-MCTS & 47 & 0 & 18 & $3.40 \%$ \\
IDABC & 47 & 0 & 17 & $4.00 \%$ \\
HVNS & 47 & 0 & 17 & $4.00 \%$ \\
PSO & 47 & 0 & 18 & $3.95 \%$ \\
GA & 47 & 0 & 17 & $4.17 \%$ \\
AIS & 47 & 0 & 16 & $4.26 \%$ \\
B\&B & 46 & $0.12 \%$ & 18 & $9.32 \%$ \\
\hline
\end{tabular}

\subsection{Comparison of Carlier and Neron's Benchmarks}

From Tables 4 and 5, only the B\&B algorithm does not obtain the optimal solution when solving the problem of $\mathrm{j} 15 \mathrm{c} 10 \mathrm{a} 5$ in the $47 \mathrm{a}$ and $\mathrm{b}$ problems. The PSO and B\&B algorithms, respectively, solve the optimal solutions of 18 problems in $30 \mathrm{c}$ and $\mathrm{d}$ problems, and the average deviation of the solutions is 3.95\% and 9.32\%, respectively. As shown in Table 6, the IDABC, HVNS and GA algorithms solve the optimal solutions for the 17 problems in the $30 \mathrm{c}$ and d problems, and the average deviation of the solutions are $4.00 \%, 4.00 \%$ and $4.17 \%$, respectively. The AIS algorithm solves the optimal solution of 16 problems in $30 \mathrm{c}$ and d problems, and the average deviation of the solution is $4.26 \%$. The ISP-MCTS algorithm proposed in this paper solves the optimal solution of 18 problems in $30 \mathrm{c}$ and d problems, and the average deviation of the solution is $3.4 \%$. Therefore, the solution quality of the ISP-MCTS algorithm for solving $\mathrm{c}$ and $\mathrm{d}$ problems is better than the other six algorithms. It can be seen from 
Tables 4 and 5 that the average CPU time of the ISP-MCTS algorithm is much smaller than that of the the PSO and B\&B algorithms. From the above comparison, the ISP-MCTS algorithm has a better solution and efficient optimization ability than the other six algorithms when solving Carlier and Neron problems.

\subsection{Comparison of Liao's Benchmarks}

From the discussion in Section 5.1, the average completion time of the three algorithms of IDABC, HVNS and PSO for solving Carlier and Neron's benchmarks is smaller than that of GA, AIS, and B\&B algorithms. Therefore, the algorithms IDABC, HVNS and PSO are selected as the comparison algorithms to solve the Liao problems in this section. In the source literature, the IDABC algorithm was coded in Visual C++ and run on an Intel Pentium 3.06 GHz PC with 2 GB RAM under the Windows 7 operating system, and each problem was tested 20 times and the termination criterion for all the tests was the computation time $100 \mathrm{~s}$. The running environment of the HVNS algorithm in the literature [20] was Intel Core i5 3.3 GHz PC with 4 GB of memory. The algorithm PSO was used to solve the Liao problems, and the algorithm limited its run time to $200 \mathrm{~s}$ and each problem runs 20 times independently. The ISP-MCTS algorithm is used to solve the Liao problems in the computer running environment proposed in this paper, and the execution time for each problem is limited to $200 \mathrm{~s}$.

As shown in Table 7, the average completion time of the ISP-MCTS solution is smaller than that of the IDABC, HVNS and PSO, and the ISP-MCTS further updates the best solution of the three problems in Liao problems. The nonparametric test is proposed, and the null hypothesis $\mathrm{H}_{0}$ assumes that $\mu_{\text {ISP-MCTS }} \geq \mu_{\text {IDABC, }}$ and the alternative hypothesis $\mathrm{H}_{1}: \mu_{\text {ISP-MCTS }}<\mu_{\text {IDABC }}$ with 0.05 significance level. Similarly, the hypotheses $\mathrm{H}_{0^{\prime}}: \mu_{\text {ISP-MCTS }} \geq \mu_{\text {HVNS }}, \mathrm{H}_{1^{\prime}}: \mu_{\text {ISP-MCTS }}<\mu_{\text {HVNS }} . \mathrm{H}_{0^{\prime \prime}}: \mu_{\text {ISP-MCTS }} \geq \mu_{\text {PSO }}$, $\mathrm{H}_{1^{\prime \prime}}: \mu_{\text {ISP-MCTS }}<\mu_{\text {PSO }}$. As shown in Table 8, the ISP-MCTS algorithm performs significantly better than the PSO and AIS algorithms, and has certain advantages in terms of solution quality and stability compared with the HVNS algorithm.

Table 7. Comparison results for the ten harder problems.

\begin{tabular}{|c|c|c|c|c|c|c|c|c|c|c|c|c|c|c|}
\hline \multirow{2}{*}{ Problem } & \multicolumn{3}{|c|}{ ISP-MCTS } & \multicolumn{5}{|c|}{ IDABC } & \multicolumn{3}{|c|}{ HVNS } & \multicolumn{3}{|c|}{ PSO } \\
\hline & Ave. & Min. & Std. & CPU & Ave. & Min. & Std. & CPU & Ave. Min. & Std. & CPU & Ave. Min. & Std. & CPU \\
\hline j30c5e1 & 463.2 & 462 & 0.81 & 14.52 & 465.2 & 463 & 1.80 & 57 & 465.41464 & 1.34 & 29.16 & 474.70471 & 1.42 & 96.16 \\
\hline j30c5e2 & 616.0 & 616 & 0 & 10.32 & 616.0 & 616 & 0 & 2 & 616.22616 & 0.32 & 11.69 & 616.25616 & 0.44 & 55.28 \\
\hline j30c5e3 & 594.7 & 593 & 0.97 & 20.37 & 596.4 & 593 & 1.70 & 49 & 598.51595 & 3.32 & 27.33 & 610.25602 & 4.70 & 64.56 \\
\hline j30c5e4 & 565.3 & 563 & 1.23 & 18.53 & 566.2 & 565 & 1.60 & 39 & 568.32566 & 1.44 & 37.00 & 577.10575 & 1.52 & 86.98 \\
\hline j30c5e5 & 600.9 & 600 & 0.77 & 17.23 & 602.0 & 600 & 1.60 & 58 & 603.75601 & 1.26 & 21.32 & 606.80605 & 1.11 & 79.84 \\
\hline j30c5e6 & 602.8 & 600 & 1.44 & 17.06 & 603.1 & 601 & 1.80 & 55 & 607.01603 & 3.32 & 33.39 & 612.50605 & 3.49 & 67.99 \\
\hline j30c5e7 & 626.4 & 626 & 0.31 & 18.23 & 626.0 & 626 & 0 & 19 & 627.34626 & 0.83 & 27.37 & 630.60629 & 0.75 & 87.18 \\
\hline j30c5e8 & 674.4 & 674 & 0.69 & 22.69 & 674.7 & 674 & 0.90 & 55 & 676.40674 & 2.23 & 31.40 & 684.20678 & 2.50 & 97.67 \\
\hline j30c5e9 & 643.1 & 642 & 0.76 & 21.37 & 643.7 & 642 & 1.00 & 67 & 645.28643 & 1.66 & 33.81 & 654.65651 & 1.87 & 83.80 \\
\hline j30c5e10 & 574.2 & 573 & 1.09 & 23.81 & 576.3 & 573 & 1.50 & 76 & 581.33577 & 3.97 & 29.72 & 599.75594 & 5.28 & 77.46 \\
\hline Ave. & 596.1 & 594.9 & 0.83 & 18.41 & 596.9 & 595.3 & 1.19 & 47.7 & 598.96596 .5 & 1.97 & 28.22 & 606.68602 .6 & 2.31 & 79.69 \\
\hline
\end{tabular}

Table 8. Comparison results for the ten harder problems.

\begin{tabular}{cccc}
\hline & ISP-MCTS and IDABC & ISP-MCTS and HVNS & ISP-MCTS and PSO \\
\hline$p$-Value & 0.12 & 0.018 & 0 \\
Significant? $(p<\mathbf{0 . 0 5})$ & NO & Yes & Yes \\
\hline
\end{tabular}

The six effective algorithms for solving HFSSP in recent years were selected for comparison with ISP-MCTS. The algorithms were: HVNS [20], IDABC [21], PSO [17], GA $A_{R}$ [2], ISA [43] and artificial bee colony with permutation $\left(\mathrm{ABC}_{\mathrm{P}}\right)$ [21]. In the computer operating environment of this study, each algorithm solved 150 problems in Section 4.1. For the comparison algorithms, HVNS, DABC, and PSO solved the same problems as reported in the literature, whereas $G A_{R}$, ISA, and $A B C_{P}$ were used to solve complex HFSSP, and the ability to solve these algorithms was verified. The above algorithms 
directly select the optimal parameter combination of the source literature. All the algorithm runtimes were limited to the maximum CPU ( $\mathrm{t}=\rho \mathrm{mn} \mathrm{s})$ time, and $\rho$ is: 10, 20, 30. All the above algorithms are programmed in MATLAB 2016a, and each of the 150 examples is run independently for 10 times, and the average of 10 running results is taken as the solution of each example. When $\rho=30$, the best solution $C_{\min }$ found by any algorithm is used to calculate the relative percentage increase (RPI). The results are shown in Tables 9-11.

Table 9. Computational results of the algorithms $(\rho=10)$.

\begin{tabular}{cccccccc}
\hline Problem & ISP-MCTS & IDABC & HVNS & PSO & GA $_{\mathbf{R}}$ & ISA & ABC $_{\mathbf{p}}$ \\
\hline $20 \times 4$ & 0.03 & 0.03 & 0.31 & 0.71 & 1.01 & 0.68 & 0.38 \\
$20 \times 6$ & 0.12 & 0.11 & 0.24 & 0.77 & 1.27 & 0.78 & 0.69 \\
$20 \times 8$ & 0.33 & 0.34 & 0.61 & 1.03 & 1.21 & 1.18 & 0.82 \\
$40 \times 4$ & 0.03 & 0.03 & 0.22 & 0.31 & 0.52 & 0.34 & 0.49 \\
$40 \times 6$ & 0.04 & 0.03 & 0.15 & 0.22 & 0.71 & 0.29 & 0.77 \\
$40 \times 8$ & 0.17 & 0.18 & 0.66 & 0.98 & 1.39 & 1.12 & 1.13 \\
$60 \times 4$ & 0.11 & 0.13 & 0.11 & 0.33 & 0.51 & 0.35 & 0.48 \\
$60 \times 6$ & 0.16 & 0.16 & 0.23 & 0.41 & 0.61 & 0.47 & 0.55 \\
$60 \times 8$ & 0.21 & 0.25 & 0.42 & 0.67 & 1.10 & 0.71 & 1.04 \\
$80 \times 4$ & 0.06 & 0.06 & 0.11 & 0.20 & 0.35 & 0.21 & 0.33 \\
$80 \times 6$ & 0.20 & 0.21 & 0.24 & 0.46 & 0.65 & 0.52 & 0.60 \\
$80 \times 8$ & 0.12 & 0.12 & 0.33 & 0.55 & 0.61 & 0.32 & 0.52 \\
$100 \times 4$ & 0.00 & 0.01 & 0.03 & 0.05 & 0.18 & 0.05 & 0.11 \\
$100 \times 6$ & 0.05 & 0.05 & 0.10 & 0.23 & 0.41 & 0.27 & 0.38 \\
$100 \times 8$ & 0.09 & 0.11 & 0.18 & 0.51 & 0.62 & 0.35 & 0.57 \\
Ave. & 0.115 & 0.121 & 0.263 & 0.495 & 0.743 & 0.509 & 0.591 \\
\hline
\end{tabular}

Table 10. Computational results of the algorithms $(\rho=20)$.

\begin{tabular}{cccccccc}
\hline Problem & ISP-MCTS & IDABC & HVNS & PSO & GA $_{\mathbf{R}}$ & ISA & ABC $_{\mathbf{p}}$ \\
\hline $20 \times 4$ & 0.02 & 0.01 & 0.27 & 0.62 & 0.88 & 0.57 & 0.29 \\
$20 \times 6$ & 0.04 & 0.06 & 0.16 & 0.68 & 1.03 & 0.66 & 0.57 \\
$20 \times 8$ & 0.21 & 0.23 & 0.47 & 0.89 & 0.96 & 1.01 & 0.44 \\
$40 \times 4$ & 0.02 & 0.03 & 0.15 & 0.24 & 0.41 & 0.29 & 0.36 \\
$40 \times 6$ & 0.01 & 0.02 & 0.11 & 0.15 & 0.56 & 0.17 & 0.51 \\
$40 \times 8$ & 0.10 & 0.13 & 0.53 & 0.73 & 1.15 & 1.01 & 0.99 \\
$60 \times 4$ & 0.05 & 0.07 & 0.09 & 0.21 & 0.47 & 0.31 & 0.42 \\
$60 \times 6$ & 0.06 & 0.10 & 0.17 & 0.33 & 0.48 & 0.36 & 0.51 \\
$60 \times 8$ & 0.12 & 0.13 & 0.31 & 0.42 & 0.92 & 0.62 & 0.93 \\
$80 \times 4$ & 0.02 & 0.02 & 0.08 & 0.11 & 0.29 & 0.18 & 0.31 \\
$80 \times 6$ & 0.09 & 0.13 & 0.17 & 0.23 & 0.65 & 0.46 & 0.48 \\
$80 \times 8$ & 0.05 & 0.09 & 0.25 & 0.33 & 0.47 & 0.28 & 0.42 \\
$100 \times 4$ & 0.00 & 0.00 & 0.02 & 0.03 & 0.13 & 0.03 & 0.09 \\
$100 \times 6$ & 0.02 & 0.02 & 0.07 & 0.11 & 0.28 & 0.16 & 0.31 \\
$100 \times 8$ & 0.06 & 0.06 & 0.21 & 0.39 & 0.46 & 0.31 & 0.48 \\
Ave. & 0.058 & 0.073 & 0.204 & 0.365 & 0.609 & 0.428 & 0.474 \\
\hline
\end{tabular}


Table 11. Computational results of the algorithms $(\rho=30)$.

\begin{tabular}{cccccccc}
\hline Problem & ISP-MCTS & IDABC & HVNS & PSO & GA $_{\mathbf{R}}$ & ISA & ABCp \\
\hline $20 \times 4$ & 0.00 & 0.00 & 0.15 & 0.43 & 0.76 & 0.49 & 0.22 \\
$20 \times 6$ & 0.03 & 0.03 & 0.12 & 0.51 & 0.97 & 0.58 & 0.52 \\
$20 \times 8$ & 0.17 & 0.21 & 0.29 & 0.77 & 0.82 & 0.91 & 0.41 \\
$40 \times 4$ & 0.00 & 0.01 & 0.11 & 0.12 & 0.33 & 0.21 & 0.27 \\
$40 \times 6$ & 0.01 & 0.01 & 0.09 & 0.11 & 0.41 & 0.14 & 0.42 \\
$40 \times 8$ & 0.06 & 0.09 & 0.35 & 0.62 & 0.95 & 0.75 & 0.86 \\
$60 \times 4$ & 0.02 & 0.02 & 0.06 & 0.17 & 0.36 & 0.26 & 0.36 \\
$60 \times 6$ & 0.04 & 0.05 & 0.13 & 0.31 & 0.41 & 0.27 & 0.44 \\
$60 \times 8$ & 0.05 & 0.06 & 0.18 & 0.37 & 0.75 & 0.45 & 0.74 \\
$80 \times 4$ & 0.00 & 0.00 & 0.07 & 0.09 & 0.24 & 0.11 & 0.26 \\
$80 \times 6$ & 0.03 & 0.05 & 0.14 & 0.18 & 0.55 & 0.32 & 0.37 \\
$80 \times 8$ & 0.05 & 0.05 & 0.16 & 0.27 & 0.43 & 0.19 & 0.34 \\
$100 \times 4$ & 0.00 & 0.00 & 0.02 & 0.03 & 0.09 & 0.01 & 0.08 \\
$100 \times 6$ & 0.01 & 0.01 & 0.06 & 0.09 & 0.26 & 0.11 & 0.28 \\
$100 \times 8$ & 0.04 & 0.04 & 0.19 & 0.32 & 0.35 & 0.18 & 0.41 \\
Ave. & 0.034 & 0.042 & 0.141 & 0.293 & 0.512 & 0.332 & 0.399 \\
\hline
\end{tabular}

As shown in Tables 9-11, the ISP-MCTS solution quality is better than that of the other six algorithms. When $\rho=10$, the average deviation of ISP-MCTS in the seven algorithms is the smallest, and the value is 0.115 . The average deviation of the ISP-MCTS algorithm is less than IDABC (0.121), HVNS (0.263), PSO (0.495), GA $A_{R}(0.743)$, ISA (0.509), and $A B C_{P}(0.591)$. Therefore, the solution quality of the ISP-MCTS algorithm is better than the other six algorithms. When $\rho=20$ and $\rho=30$, the average deviation of all algorithms is improved through comparing to $\rho=10$. At the same time, the ISP-MCTS algorithm still has the smallest deviation among all algorithms, so the validity of the ISP-MCTS algorithm can be verified.

Here, a multi-factor analysis of variance was used to further compare the seven algorithms, and the algorithm type and $\rho$ were taken as factors. From the mean graph and the two-factor Tukey HSD test (95\% confidence interval) as shown in Figure 3, the ISP-MCTS algorithm is significantly better than the HVNS, PSO, GA, ISA, and $\mathrm{ABC}_{\mathrm{P}}$ algorithms at different CPU times. According to the above average variance value, the superiority of the ISP-MCTS algorithm in solving the HFSSP problem was verified.

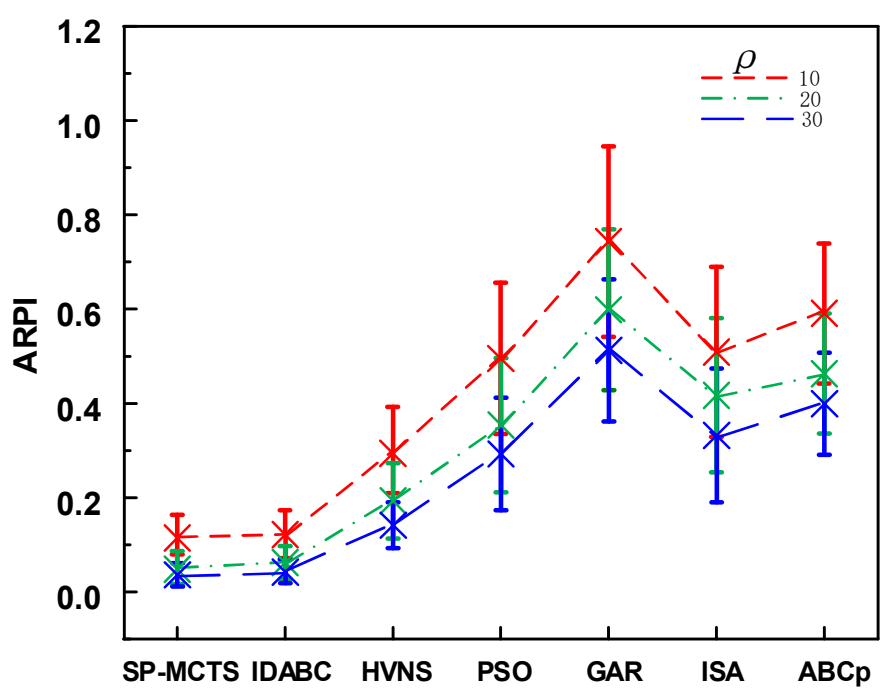

Figure 3. The means plot and 95\% Tukey HSD confidence intervals for the interaction between the algorithms and the allowed CPU time. 


\section{Conclusions}

1. This paper analyzed the HFSSP operation mechanism, and proposed a tree search algorithm based on SP-MCTS to solve HFSSP. In order to solve the problem of HFSSP by SP-MCTS algorithm, the three steps of selection, expansion and simulation were improved respectively. The improvement of the algorithm included the selection blending standard deviation, the single-branch expansion strategy and the 4-Rule policy simulation. The results show that these improvements can quickly and accurately locate high-potential branches, and obtain the optimal solution.

2. The 4-Rule simulation policy was selected based on a comparative analysis of the problems, and the 4-Rule can give an accurate evaluation of the leaf nodes during the search process, thus improving the search efficiency of the algorithm. According to the magnitude of the search, the combination of the parameters $C$ and $D$ was determined to ensure the convergence speed of the algorithm.

3. The experimental results of the standard problems show that the ISP-MCTS algorithm is effective, and the optimal solutions of 47 class a and class $b$ problems can be obtained at $100 \%$. The ISP-MCTS algorithm solved the optimal solution of 18 problems in the $\mathrm{c}$ and $\mathrm{d}$ problems, and the average deviation was $3.4 \%$, which was lower than the other five comparison algorithms. This proves that the ISP-MCTS algorithm has certain advantages in solving the HFSSP problems. According to the statistical analysis results of each algorithm to solve the Liao problems, the ISP-MCTS performance is obviously better than the HVNS and PSO algorithms, and it has a certain superiority in solution quality and stability compared with the IDABC algorithm.

4. Through the comparison of large-scale random problems, it shows that the ISP-MCTS algorithm is superior to the comparison algorithms in terms of CPU time, solution quality and stability.

Author Contributions: J.G. introduced the concepts of this paper and collected the corresponding references. P.Z. and J.G. conducted the experimental work, took part in the data acquisition. T.Y., Z.C. and Y.S. participated in the research design, data acquisition, data analysis and manuscript preparation. Finally, J.G. analyzed and interpreted the data, B.S. and P.Z. reviewed this paper and made constructive comments. All authors read and agreed to the published version of the manuscript.

Funding: This paper was supported by National Science and Technology Major Project of China (No.2018ZX04005001) and the Major Projects of Aero Engines and Gas Turbines (grant No. 2017-VII-0002-0095).

Conflicts of Interest: The authors declare no conflict of interest.

\section{References}

1. Johnson, S.M. Optimal two- and three-stage production schedules with setup times included. Nav. Res. Logist. Q. 1954, 1, 61-68. [CrossRef]

2. Ruiz, R.; Maroto, C. A genetic algorithm for hybrid flowshops with sequence dependent setup times and machine eligibility. Eur. J. Oper. Res. 2006, 169, 781-800. [CrossRef]

3. Gupta, J.N.D. Two-Stage, Hybrid Flowshop Scheduling Problem. J. Oper. Res. Soc. 1988, 39, 359-364. [CrossRef]

4. Wang, J.; Zhang, Y.; Liu, Y.; Wu, N. Multi-agent and Bargaining-game-based Real-time Scheduling for Internet of Things-enabled Flexible Job Shop. IEEE IoT J. 2018, 6, 1.

5. Ribas, I.; Leisten, R.; Framinan, J.M. Review and classification of hybrid flow shop scheduling problems from a production system and a solutions procedure perspective. Comput. Oper. Res. 2010, 37, 1439-1454. [CrossRef]

6. Arthanary, T.S.; Ramaswamy, K.G. An extension of two machine sequencing problems. Oper. Res. 1971, 8 , $10-22$.

7. Portmann, M.-C.; Vignier, A.; Dardilhac, D.; Dezalay, D. Branch and bound crossed with GA to solve hybrid flowshops. Eur. J. Oper. Res. 1998, 107, 389-400. [CrossRef]

8. Néron, E.; Baptiste, P.; Gupta, J.N. Solving hybrid flow shop problem using energetic reasoning and global operations. Omega 2001, 29, 501-511. [CrossRef] 
9. Gupta, J.N.D.; Tunc, E.A. Minimizing tardy jobs in a two-stage hybrid flow shop. Int. J. Prod. Res. 1998, 36, 2397-2417. [CrossRef]

10. Kahraman, C.; Engin, O.; Kaya, I.; Öztürk, R.E. Multiprocessor task scheduling in multistage hybrid flow-shops: A parallel greedy algorithm approach. Appl. Soft Comput. 2010, 10, 1293-1300. [CrossRef]

11. Lin, H.-T.; Liao, C.-J. A case study in a two-stage hybrid flow shop with setup time and dedicated machines. Int. J. Prod. Econ. 2003, 86, 133-143. [CrossRef]

12. Fakhrzad, M.; Heydari, M. A Heuristic Algorithm for Hybrid Flow-Shop Production Scheduling to Minimize the Sum of the Earliness and Tardiness Costs. J. Chin. Inst. Ind. Eng. 2008, 25, 105-115. [CrossRef]

13. Paternina-Arboleda, C.D.; Montoya-Torres, J.R.; Acero-Dominguez, M.J.; Herrera-Hernandez, M.C. Scheduling jobs on a k-stage flexible flow-shop. Ann. Oper. Res. 2007, 164, 29-40. [CrossRef]

14. Nowicki, E.; Smutnicki, C. The flow shop with parallel machines: A tabu search approach. Eur. J. Oper. Res. 1998, 106, 226-253. [CrossRef]

15. Alaykýran, K.; Engin, O.; Döyen, A. Using ant colony optimization to solve hybrid flow shop scheduling problems. Int. J. Adv. Manuf. Technol. 2007, 35, 541-550. [CrossRef]

16. Kahraman, C.; Engin, O.; Kaya, I.; Yilmaz, M.K. An application of effective genetic algorithms for solving hybrid flow shop scheduling problems. Int. J. Intell. Syst. 2008, 1, 134-147. [CrossRef]

17. Liao, C.J.; Tjandradjaj, E.; Chung, T.P. An approach using particle swarm optimization and bottleneck heuristic to solve flowshop scheduling problem. Appl. Soft Comput. 2012, 12, 1755-1764. [CrossRef]

18. Marichelvam, M.K.; Prabaharan, T.; Yang, X.-S. Improved cuckoo search algorithm for hybrid flow shop scheduling problems to minimize makespan. Appl. Soft Comput. 2014, 19, 93-101. [CrossRef]

19. Pan, Q.-K.; Wang, L.; Li, J.-Q.; Duan, J.-H. A novel discrete artificial bee colony algorithm for the hybrid flowshop scheduling problem with makespan minimisation. Omega 2014, 45, 42-56. [CrossRef]

20. Li, J.-Q.; Pan, Q.-K.; Wang, F.-T. A hybrid variable neighborhood search for solving the hybrid flow shop scheduling problem. Appl. Soft Comput. 2014, 24, 63-77. [CrossRef]

21. Cui, Z.; Gu, X. An improved discrete artificial bee colony algorithm to minimize the makespan on hybrid flow shop problems. Neurocomputing 2015, 148, 248-259. [CrossRef]

22. Komaki, G.; Teymourian, E.; Kayvanfar, V. Minimising makespan in the two-stage assembly hybrid flow shop scheduling problem using artificial immune systems. Int. J. Prod. Res. 2015, 54, 1-21. [CrossRef]

23. Zhang, X.Y.; Chen, L. A re-entrant hybrid flow shop scheduling problem with machine eligibility constraints. Int. J. Prod. Res. 2017, 56,1-13. [CrossRef]

24. Niu, Q.; Zhou, T.; Ma, S. A quantum-inspired immune algorithm for hybrid flowshop with makespan criterion. J. Univ. Comput. Sci. 2009, 15, 765-785.

25. Engin, O.; Döyen, A. A new approach to solve hybrid flow shop scheduling problems by artificial immune system. Futur. Gener. Comput. Syst. 2004, 20, 1083-1095. [CrossRef]

26. Ramanan, T.R.; Sridharan, R.; Shashikant, K.S.; Haq, A.N. An artificial neural network based heuristic for flow shop scheduling problems. J. Intell. Manuf. 2009, 22, 279-288. [CrossRef]

27. Izonin, I.; Tkachenko, R.; Kryvinska, N.; Tkachenko, P.; Greguš ml., M. Multiple Linear Regression Based on Coefficients Identification Using Non-iterative SGTM Neural-like Structure. In Advances in Computational Intelligence; Springer: Cham, Switzerland, 2019.

28. Tkachenko, R.; Izonin, I. Model and Principles for the Implementation of Neural-Like Structures Based on Geometric Data Transformations; Springer Science and Business Media LLC: Heidelberg, Germany, 2018; pp. 578-587.

29. Gupta, J.N.D.; Majumder, A.; Laha, D. Flowshop scheduling with artificial neural networks. J. Oper. Res. Soc. 2019, 1-19. [CrossRef]

30. Zhao, F.; Qin, S.; Zhang, Y.; Ma, W.; Zhang, C.; Song, H. A hybrid biogeography-based optimization with variable neighborhood search mechanism for no-wait flow shop scheduling problem. Expert Syst. Appl. 2019, 126, 321-339. [CrossRef]

31. Powley, E.J.; Cowling, P.I.; Whitehouse, D. Information capture and reuse strategies in Monte Carlo Tree Search, with applications to games of hidden information. Artif. Intell. 2014, 217, 92-116. [CrossRef]

32. Chaslot, G.; De, J.S.; Saito, J.T. Monte-Carlo tree search in production management problems. In Proceedings of the 18th Belgium-Netherlands Conference on Artificial Intelligence, Namur, Belgium, 5-6 October 2006; Volume 2, pp. 91-98. 
33. Wu, T.-Y.; Wu, I.-C.; Liang, C.-C. Multi-objective Flexible Job Shop Scheduling Problem Based on Monte-Carlo Tree Search. In Proceedings of the 2013 Conference on Technologies and Applications of Artificial Intelligence, Taipei, Taiwan, 6-8 December 2013; pp. 73-78. [CrossRef]

34. Chou, J.-J.; Liang, C.-C.; Wu, H.-C.; Wu, I.-C.; Wu, T.-Y.; Jen-Jai, C.; Chao-Chin, L.; Hung-Chun, W.; I-Chen, W.; Tung-Ying, W. A new MCTS-based algorithm for multi-objective flexible job shop scheduling problem. In Proceedings of the 2015 Conference on Technologies and Applications of Artificial Intelligence (TAAI), Tainan, Taiwan, 20-22 November 2015; Institute of Electrical and Electronics Engineers (IEEE): Piscataway, NJ, USA, 2015; pp. 136-141.

35. Furuoka, R.; Matsumoto, S. Worker's knowledge evaluation with single-player Monte Carlo tree search for a practical reentrant scheduling problem. Artif. Life Robot. 2016, 22, 130-138. [CrossRef]

36. Schadd, M.P.D.; Winands, M.H.M.; Herik, J.V.D.; Chaslot, G.; Uiterwijk, J.W.H.M. Single-Player Monte-Carlo Tree Search. Computers and Games; Springer: Berlin/Heidelberg, Germany, 2008.

37. Schadd, M.P.; Winands, M.H.M.; Tak, M.J.; Uiterwijk, J.W.H.M. Single-player Monte-Carlo tree search for SameGame. Knowl. Based Syst. 2012, 34, 3-11. [CrossRef]

38. Shimpei, M.; Noriaki, H.; Kyohei, I. Evaluation of Simulation Strategy on Single-Player Monte-Carlo Tree Search and its Discussion for a Practical Scheduling Problem. Lect. Notes Eng. Comput. Sci. 2010, 1, 2182.

39. Sylvain, G.; David, S. Monte- Carlo tree search and rapid action value estimation in computer Go. Artific. Intell. 2011, 175, 1856-1875.

40. Hazeghi, K.; Puterman, M.L. Markov Decision Processes: Discrete Stochastic Dynamic Programming. J. Am. Stat. Assoc. 1995, 90, 392. [CrossRef]

41. Boutilier, C. Knowledge Representation for Stochastic Decision Processes: Artificial Intelligence Today; Springer Science and Business Media LLC: Heidelberg, Germany, 1999; Volume 1600, pp. 111-152.

42. Carlier, J.; Néron, E. An Exact Method for Solving the Multi-Processor Flow-Shop. RAIRO Oper. Res. 2000, 34, 1-25. [CrossRef]

43. Naderi, B.; Zandieh, M.; Balagh, A.K.G.; Roshanaei, V. An improved simulated annealing for hybrid flowshops with sequence-dependent setup and transportation times to minimize total completion time and total tardiness. Expert Syst. Appl. 2009, 36, 9625-9633. [CrossRef]

(C) 2020 by the authors. Licensee MDPI, Basel, Switzerland. This article is an open access article distributed under the terms and conditions of the Creative Commons Attribution (CC BY) license (http://creativecommons.org/licenses/by/4.0/). 